\title{
NEW METHOD FOR ANALYSIS OF LIQUID CRYSTAL ELASTICITY
}

\author{
A. WALCZAK \\ Institute of Applied Physics, Military University of Technology \\ Kaliskiego 2, 01-489 Warsaw, Poland
}

(Received July 15, 1998; in final form September 22, 1998)

\begin{abstract}
Orientational elastic constant $k_{11}, k_{33}$ and $k_{13}$ of liquid crystals are determined by a new way. Liquid crystalline waveguide is applied to measure refractive index profile across the waveguide layer. Results of the measurements allow one to find local director field within the liquid crystalline layer. Director field is applied to transform the Frank-Oseen equations into a form of an overdetermined algebraic system of equations with elastic constants as unknown variables. A solution of those equations has been found by the orthogonal decomposition method. Elastic constants for nematic mixture, W602, and main component of it, $6 \mathrm{CHBT}$ (4-trans-4- $n$-hexyl-cyclohexyl-isothiocyanatobenzene), have been obtained as well as anchoring energy values
\end{abstract}

PACS numbers: $61.30 . \mathrm{Gd}$

\section{Introduction}

Liquid crystal elasticity measurements are usually made by observance of the threshold response of the liquid crystalline (lc) device dependent upon voltage of the effective field acting over liquid crystal material. In Frederick's transition (FT) threshold values of electric and magnetic fields are connected with elastic constants [1]. Unfortunately accurate values $E_{\text {th }}$ or $H_{\text {th }}$ associated with FT may be only achieved if the boundary angle $\Theta_{B}$ exactly equals 0 or $\pi / 2$ and if it does not vary with field voltage [2]. Each other approach to the lc elasticity determination avoiding threshold phenomena always falls into dependence on boundary angle $[2,3]$. The aim of the article is to present a new method for elasticity analysis. The presented method does not depend upon the mentioned constraints. Bulk and surface elasticity can be examined as well as anchoring energy. The key is a direct refractive index profile measurement in the lc layer arranged as a waveguide $[4,5]$. After that, local director field is obtained. Assuming that we have measured local director field in the thin lc layer we can try to find values of elastic constants and anchoring energy as well immediately from the equation of motion. 
To avoid uncertainties, the investigations have been done for two lc nematic substances: 4-trans-4- $n$-hexyl-cyclohexyl-isothiocyanatobenzene (6CHBT) described in the literature $[2,4]$ and nematic mixture W602, in which the main component is $6 \mathrm{CHBT}$. The elastic constant $k_{13}$ has been obtained for the first time for 6CHBT similarly as all other elastic constants for W602.

\section{Director field determination}

The method for direct director field measurement has been described earlier $[5,6]$, therefore only a short review is presented here. A similar construction of double couplers has been applied in attenuated total reflection (ATR) experiments [7-15]. Differences between the presented approach and ATR method have been described earlier [16].

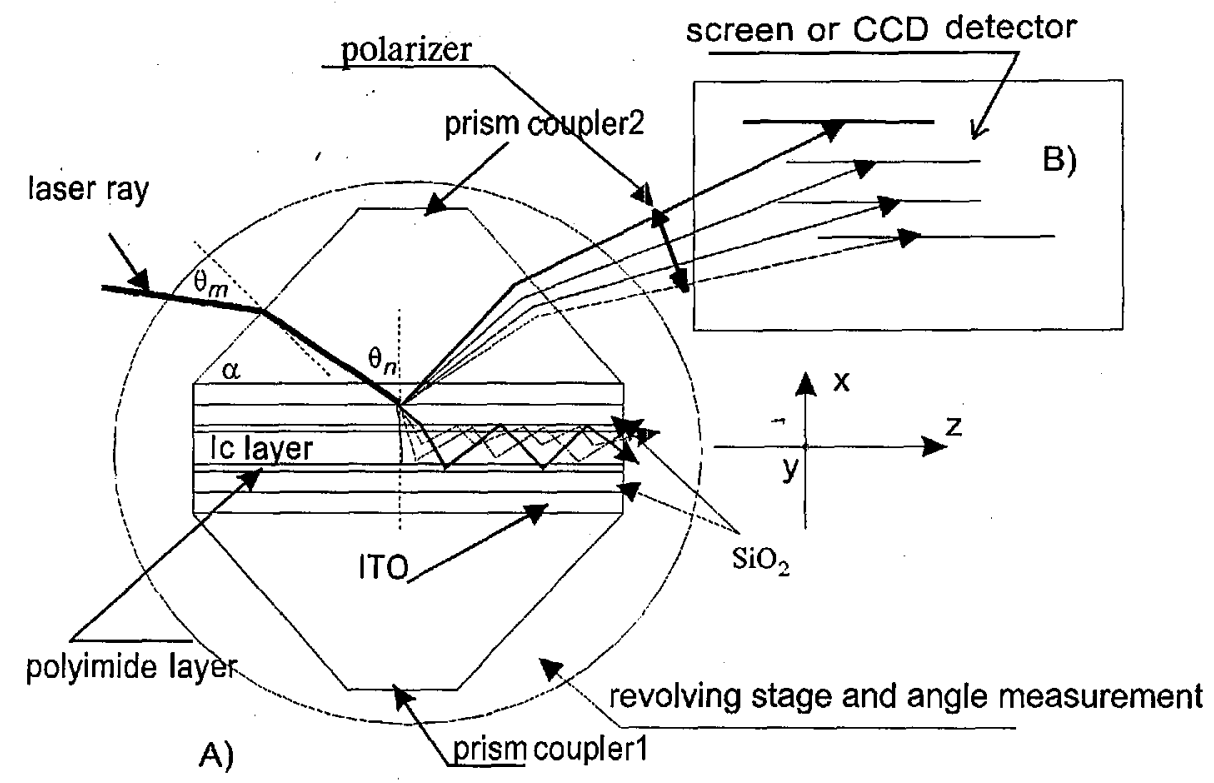

Fig. 1. Layers arrangement in the waveguide construction between the prism couplers (A) and the scheme of modal lines (B).

A pair of prism couplers is applied to obtain a modal line picture as in Fig. 1. Between the couplers the Ic layer arranged as a waveguide is placed. Refractive indices of the layers between prisms have been chosen properly to obtain a tunable Ic waveguide. Each modal line is excited during coupler rotation on revolving stage. The angle at which the observed line is excited has been precisely measured. The effective propagation constant for an excited mode $\beta=N_{m} k_{0}$ ( $k_{0}$ is the wave number in free space) can be determined from

$$
N_{m}=n_{p} \sin \left[\alpha+\arcsin \left(\frac{\sin \left(\Theta_{m}\right)}{n_{p}}\right)\right]
$$


which is obtained during modal line excitation by the measurement of $\Theta_{m}$ angle (see Fig. 1). The indication $n_{p}$ in Eq. (2.1) means the refractive index of the prism coupler.

The refractive index profile in the $l c$ waveguide is tuned by an external electric field directed perpendicularly to the waveguide plane. Modal lines are moved to a different angle position for each electric field voltage. An effective propagation constant of each mode is measured versus field voltage. The propagation constants of the modes propagating in the waveguide fulfil the following condition $[17,18]$ :

$$
2 k_{0} \int_{0}^{x_{m}}\left[n^{2}(x)-N_{m}^{2}\right]^{1 / 2} \mathrm{~d} x+2 \phi_{c a}+2 \phi_{c b}=2 \pi m,
$$

where

$$
\phi_{c a, c b}=\arctan \left[\frac{n\left(x_{m}\right)}{n_{a, b}}\right]^{\rho} \sqrt{\frac{N_{m}^{2}-n_{a, b}^{2}}{n^{2}\left(x_{m}\right)-N_{m}^{2}}} .
$$

$a$ and $b$ denote areas of optical buffer $\mathrm{ITO} / \mathrm{SiO}_{2} /$ polyimide on both the sides of the lc layer between prism couplers (ITO is indium tin oxide). For the wave polarized in the plane of incidence, $\rho$ is equal to 0 and for the wave polarized perpendicularly to that plane it is equal to 1 .

An example of the modal line that is observed in the 6CHBT tunable waveguide is shown in Fig. 2. In the waveguide electromagnetic fields of incident wave

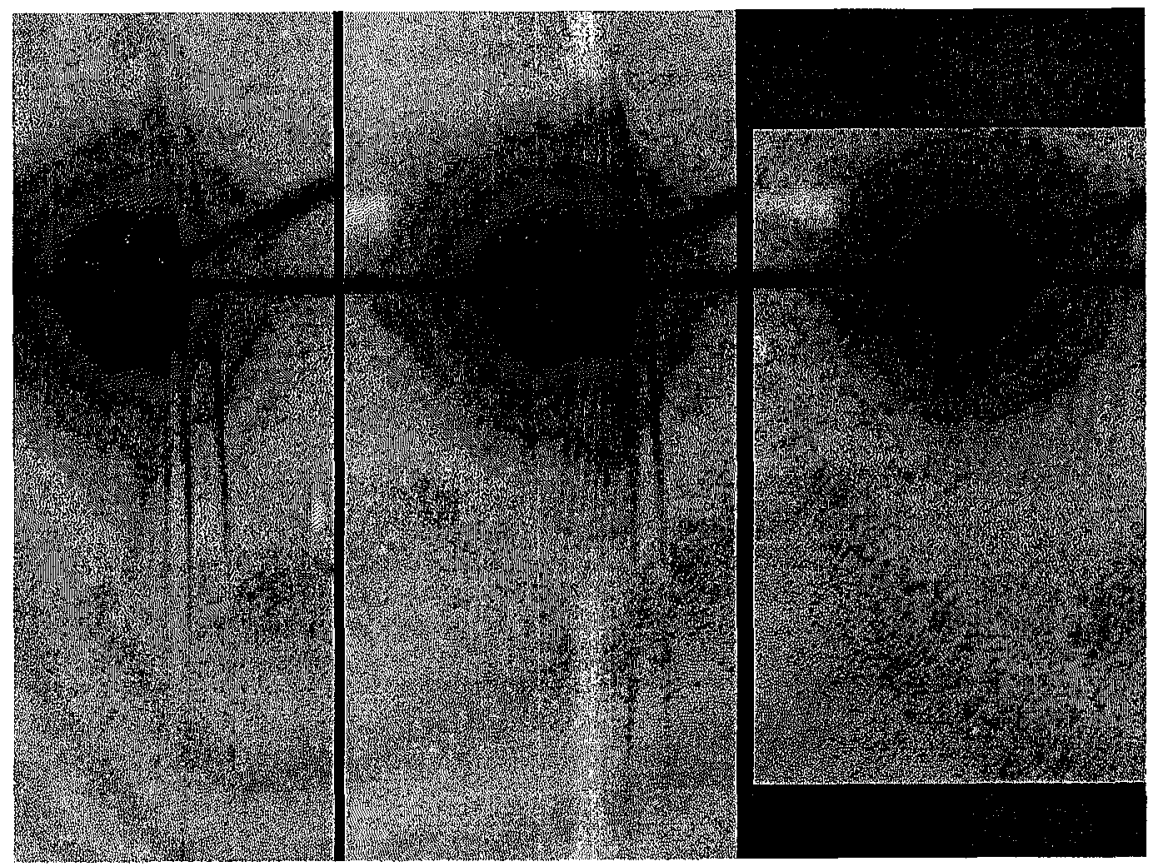

Fig. 2. Picture of modal lines from CCD camera for the tuned 6CHBT waveguide with the initially twisted lc layer in different electric fields: $1.5 \mathrm{~V}, 1.8 \mathrm{~V}$ and $2.3 \mathrm{~V}$, appropriately from left to right picture. 
and propagating modes are present [17] what can be seen in Fig. 2. The excited line intensity is much higher than other modal lines and in opposite to incident wave this line is sensitive to external field voltage.

The refractive index profile in the $l c$ waveguide is tuned by an external electric field directed perpendicularly to the waveguide plane. Modal lines are moved to a different angle position for each electric field voltage. An effective propagation constant of each mode is measured versus field voltage following formula (2.1).

The turning point $x_{m}$ is defined by $n\left(x_{m}\right)=N_{m}$, where $N_{m}$ is the effective refractive index for a mode $m$. The refractive index profile $n(x)$ is unknown. We assume for the difference $n^{2}(x)-N_{m}^{2}$ in formula (2.2) that

$$
n(x)=N_{k}+\left(\frac{N_{k-1}-N_{k}}{x_{k}-x_{k-1}}\right)\left(x_{k}-x\right)
$$

and the sum $n(x)+N_{m}$ have been replaced with

$$
\frac{\left(N_{k-1}+N_{k}\right)}{2} \text {. }
$$

Finally from (2.2) and (2.3) one obtains after receiving $(2.4,2.5)$

$$
\begin{gathered}
\sum_{k=1}^{m} \frac{1}{2\left(N_{k-1}-N_{k}\right)}\left\{\left(x_{k-1}-x_{k}\right)\left(N_{k} \sqrt{N_{k}^{2}-N_{m}^{2}}\right)\right. \\
+N_{m}^{2} \log \left[\left(N_{k}-\sqrt{N_{k}^{2}-N_{m}^{2}}\right)\left(x_{k}-x_{k-1}\right)\right]+\left(x_{k}-x_{k-1}\right)\left(N_{k-1} \sqrt{N_{k-1}^{2}-N_{m}^{2}}\right) \\
\left.-N_{m}^{2} \log \left[\left(N_{k-1}-\sqrt{N_{k-1}^{2}-N_{m}^{2}}\right)\left(x_{k}-x_{k-1}\right)\right]\right\}-\left(m+\frac{1}{2}\right) \pi=0 .
\end{gathered}
$$

The recurrence formula (2.6) and formula (2.4) are used to find $x_{k}$ and to obtain $n(x)$ from (2.2). The resulting function $n(x)$ initially linear between turning points $x_{k}$ is approximated with a polynomial and placed into (2.2). This provides a correction for $x_{0}$ and $x_{1}$ for the first two modes and the whole procedure is repeated until receiving a demanded accuracy. Examples of the refractive index profiles achieved by this procedure are illustrated in Fig. 3.

Displacements of turning points versus external field voltage illustrate the lc layer deformation for different external voltages.

From the refractive index profile by the known formula for optics of birefringent substances

$$
n_{\mathrm{eff}}(\gamma)=\frac{n_{\mathrm{e}} n_{\circ}}{\sqrt{n_{\mathrm{e}}^{2} \sin ^{2} \gamma+n_{\mathrm{o}}^{2} \cos ^{2} \gamma}},
$$

where $n_{\mathrm{e}}$ is the extraordinary refractive index and $n_{\mathrm{o}}$ is the ordinary refractive index for the examined lc substance, one can determine an angle $\gamma$ between the wave vector of a mode and local optical axis in the turning point $[5,6]$. It is possible because of $n_{\mathrm{eff}}\left(x_{k}\right)=N_{k}$ for each mode in the waveguide [18]. The position of a local optical axis is equivalent to director field in aligned lc layers. So having $n_{\text {eff }}\left(x_{k}\right)$ one obtains $\Theta\left(x_{k}\right)$. To obtain it from (2.7) a regime for clear TM and TE modes in the waveguide have to be imposed [6]. 

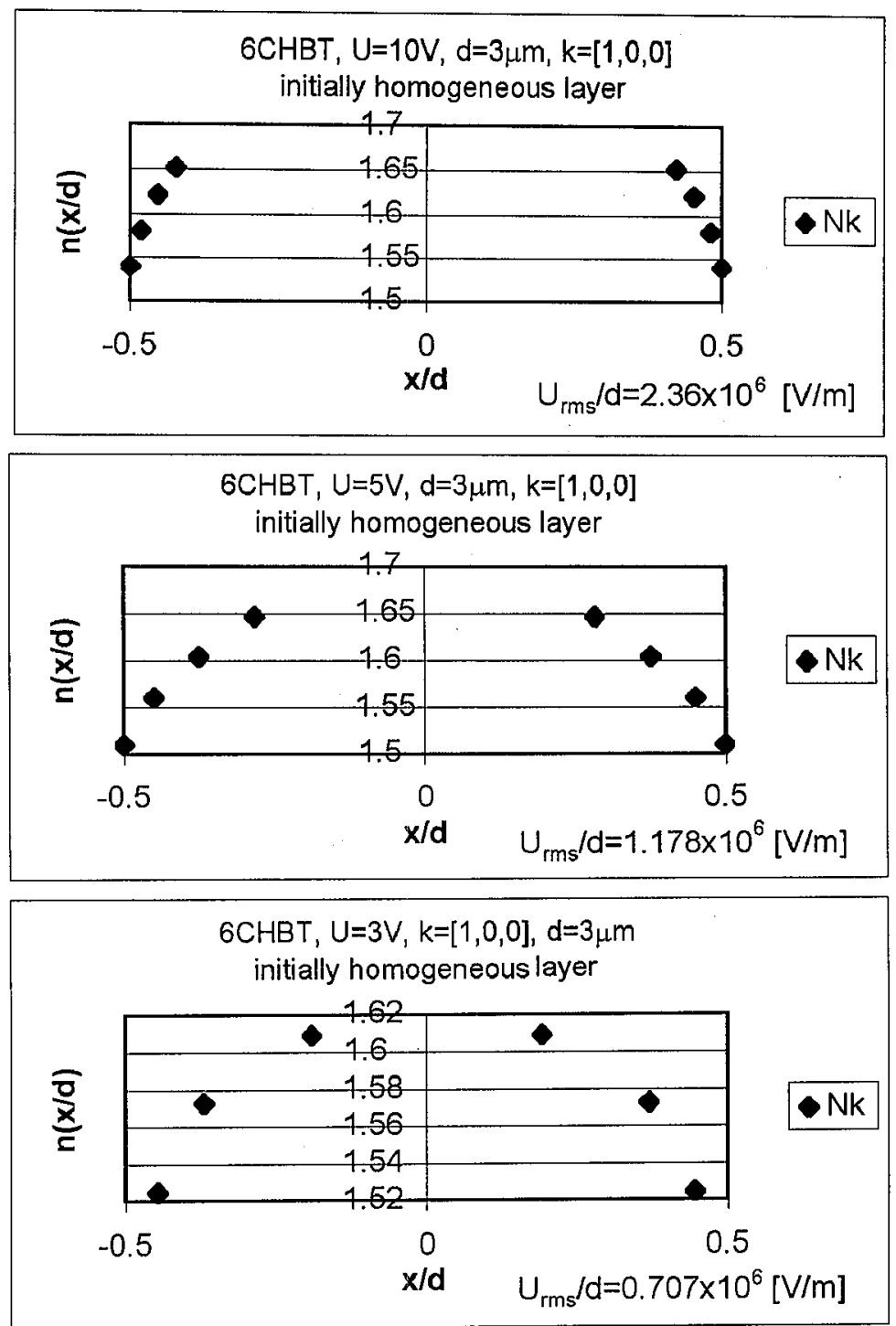

Fig. 3. Refractive index profile $n\left(x_{k}\right)$ in turning points obtained in the 6CHBT tuned waveguide for different external electric field voltages.

\section{Analysis of elastic lc layer properties}

Homogeneously an aligned layer has been arranged as a lc waveguide. Equations and boundary condition are of the following form [1]:

$$
\begin{aligned}
& \left(k_{11} \cos ^{2} \theta+k_{33} \sin ^{2} \theta\right) \frac{\partial^{2} \theta}{\partial z^{2}}+\left(k_{33}-k_{11}\right) \sin \theta \cos \theta\left(\frac{\partial \theta}{\partial z}\right)^{2} \\
& +\varepsilon_{0} \varepsilon_{\mathrm{eff}}(z) E^{2} \sin \theta \cos \theta=0
\end{aligned}
$$




$$
k_{33} \cos ^{2} \theta\left|\frac{\partial \theta}{\partial z}\right|+k_{11} \sin ^{2} \theta\left|\frac{\partial \theta}{\partial z}\right|-\frac{\partial g_{R s}(\theta)}{\partial \theta}=0, \quad z= \pm \frac{d}{2},
$$

where [1]

$$
\begin{aligned}
& \varepsilon_{\mathrm{eff}}(z)=\varepsilon_{\perp}+\Delta \varepsilon n(z) \cdot n(z), \\
& \Delta \varepsilon=\varepsilon_{\|}-\varepsilon_{\perp}, \\
& n(z)=(\cos \theta(z), 0, \sin \theta(z)) .
\end{aligned}
$$

The angles in formulae (3.1), (3.2) and (3.3) are as in Fig. 4.

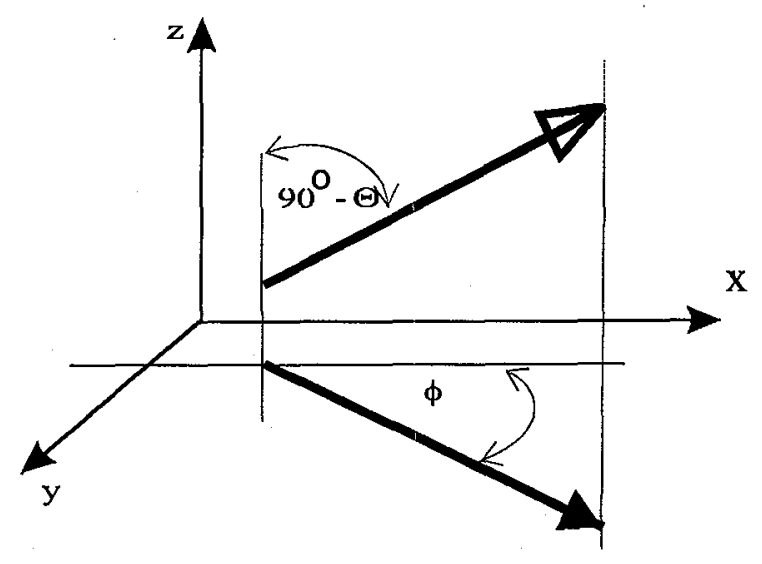

Fig. 4. The angles chosen for director description.

The orientation of the director within the waveguide layer is used in the Frank-Oseen equations to describe the lc deformation in an external electric field. Having the profile $\Theta(x)$ measured from refractive index profile (RIP) each equation can be transformed into algebraic one for a number of points across the layer. In this algebraic system of equations the unknown variables are elastic constants $k_{11}, k_{33}$. Electric permittivity of the examined liquid crystals is additive data from the literature $[19,20]$. A number of equations exceeded the number of variables. In other words the differential equations have been replaced by the algebraic problem with sufficient data to overdetermine a solution. The problem has been solved numerically by the orthogonal decomposition method [21, 22].

The director field profiles have been measured for different external voltages and proper results for elastic constants are illustrated in Table I.

Measurements of the elastic constants of 6CHBT have been reported earlier and are compared with the present results in Table II.

The obtained values of $k_{11}$ and $k_{33}$ were applied in the boundary conditions (3.2) to find the surface torque. Results are shown in Fig. 5.

The nematic liquid crystal (NLC) can be oriented by solid substrates along the well-defined directions $\boldsymbol{e}_{0}$, called easy directions. The interaction lc-solid surface is described by the function $g_{s}$ called anchoring energy. If the nematic scalar 
TABLE I

Elastic constants obtained for different field voltages.

\begin{tabular}{c|c|c|c|c}
\hline \multirow{2}{*}{$\begin{array}{c}\text { Voltage } \\
\text { [V] }\end{array}$} & \multicolumn{2}{|c|}{6 CHBT } & \multicolumn{2}{c}{ W602 } \\
\cline { 2 - 5 } & $\begin{array}{c}k_{11} \\
{\left[10^{-12} \mathrm{~N}\right]}\end{array}$ & $\begin{array}{c}k_{33} \\
{\left[10^{-12} \mathrm{~N}\right]}\end{array}$ & $\begin{array}{c}k_{11} \\
{\left[10^{-12} \mathrm{~N}\right]}\end{array}$ & $\begin{array}{c}k_{33} \\
{\left[10^{-12} \mathrm{~N}\right]}\end{array}$ \\
\hline 2.1 & 6.0 & 7.1 & 13.0 & 15.95 \\
2.3 & 6.45 & 6.4 & 12.7 & 16.2 \\
2.4 & 6.0 & 6.9 & 12.45 & 14.7
\end{tabular}

TABLE II

Comparison of the results referred earlier in the literature for 6CHBT.

\begin{tabular}{c|c|c|c|c|c}
\hline \hline \multicolumn{5}{c}{ Elastic constants $\left[10^{-12} \mathrm{~N}\right]$} \\
\hline \multicolumn{3}{c}{ From [19] } & \multicolumn{2}{c}{ From [2] } & \multicolumn{2}{c}{ This work } \\
\hline$k_{11}$ & $k_{33}$ & $k_{11}$ & $k_{33}$ & $k_{11}$ & $k_{33}$ \\
\hline 6.71 & 7.38 & 6.50 & 9.5 & 6.15 & 6.8
\end{tabular}

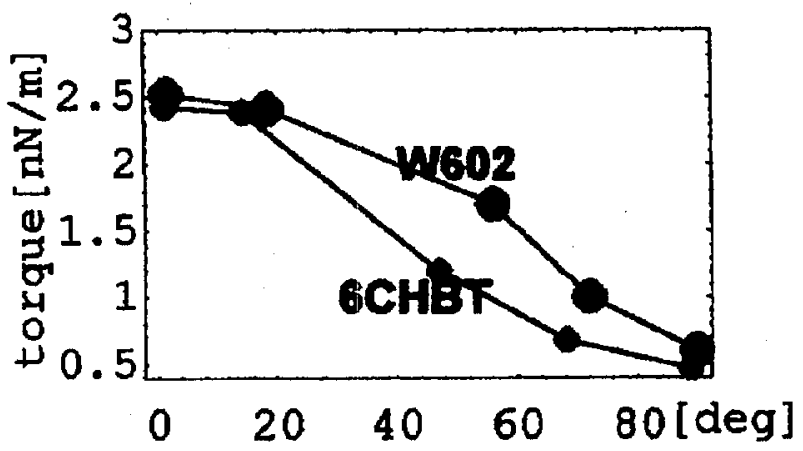

Fig. 5. The surface torque versus tilt angle on the lc layer boundary in the initially homogeneous layer.

order parameter $S$ is assumed uniform then the monostable anchoring energy can be written in the Rapini-Papoular (RP) form [23]

$$
g_{s}=-\frac{1}{2} W_{s}\left(n e_{0}\right)^{2} \text {. }
$$

For stronger anchoring deviations from this formula have been reported [24]. Some time ago Yokoyama suggested that a spatial variation of the elastic constant is equivalent to a surface energy [25]. Other authors reconsidered that problem too [26]. Faeti [27] showed that the subsurface elastic effect is fully equivalent to renormalization of the anchoring energy. He conclude that one can disregard the $k_{13}$ elastic constant in the expression of the bulk free energy and account it for its effect by defining a new effective anchoring potential. A surface torque in Eq. (3.2) 
following that conclusion is a result of the lc molecules anchoring and lc surface interactions as well. Taking into account the surface elasticity one must change the boundary condition (3.2) to the form [28]

$$
\begin{aligned}
& k_{11} \cos ^{2} \theta \mid \frac{\partial \theta}{\partial z} \\
& k_{11} \cos ^{2} \theta\left|+k_{33} \sin ^{2} \theta\right| \frac{\partial \theta}{\partial z}\left|+k_{33} \sin ^{2} \theta\right| \frac{\partial \theta}{\partial z} \mid+\frac{\partial}{\partial \theta} g_{s}(\theta)+k_{13} \frac{\partial \theta}{\partial z} \sin 2 \theta=0, \quad z=\frac{d}{2}, \\
& +\frac{\partial}{\partial \theta} g_{s}(\theta)-k_{13} \frac{\partial \theta}{\partial z} \sin 2 \theta=0, \quad z=-\frac{d}{2} .
\end{aligned}
$$

So we can take as a surface torque the following renormalized function:

$$
\frac{\partial g_{R s}}{\partial \theta}=-W_{s} \sin 2 \theta-\frac{1}{2 d} k_{13} \sin 2 \theta \frac{\partial \theta}{\partial \xi}
$$

where $\xi=x d$ and $d$ is a layer thickness. Anchoring energy $g_{s}$ has been assumed in the Rapini-Papoular form. While knowing $k_{11}$ and $k_{33}$, from the boundary conditions (3.2) one obtains the values of surface torque versus director tilt. The fitting of the function (3.5) by the least square method to those experimental values results in $W_{s}$ and $k_{13}$ placed in Table III. The sign of $k_{13}$ remains in accordance with the predictions of Nehring and Saupe [29], who introduced it into the Frank-Oseen equations. So there exists a correspondence to microscopic models.

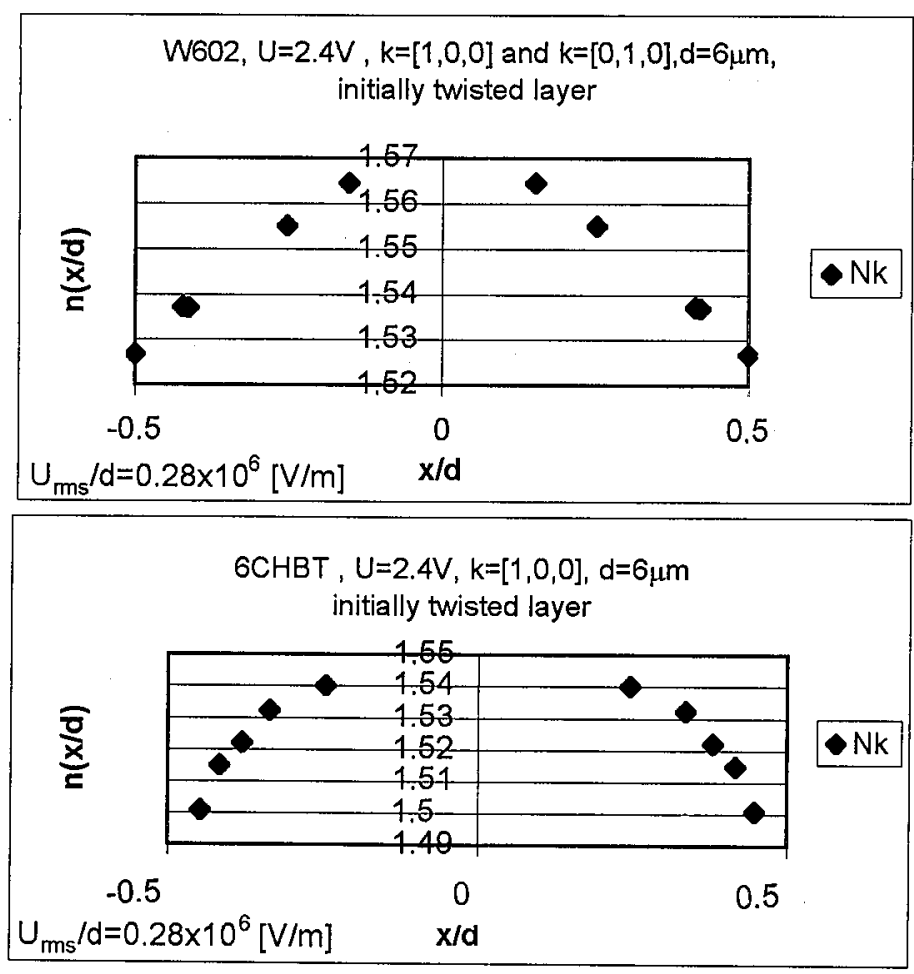

Fig. 6. Refractive index profiles determined in turning points for the $6 \mathrm{CHBT}$ and $\mathrm{W} 602$ initially twisted layers. 
TABLE III

Surface elastic constant and anchoring energy in the examined lc materials.

\begin{tabular}{c|c|c|c|c}
\hline & \multicolumn{2}{|c|}{$k_{13}\left[10^{-12} \mathrm{~N}\right]$} & \multicolumn{2}{c}{$W_{s}\left[\mathrm{~J} / \mathrm{m}^{2}\right]$} \\
\cline { 2 - 5 } & $d=3 \mu \mathrm{m}$ & $d=6 \mu \mathrm{m}$ & $d=3 \mu \mathrm{m}$ & $d=6 \mu \mathrm{m}$ \\
\hline W602 & -5.56 & -6.23 & $1.13 \times 10^{-7}$ & $2.45 \times 10^{-7}$ \\
$6 \mathrm{CHBT}$ & -4.31 & -5.54 & $18.4 \times 10^{-7}$ & $14.2 \times 10^{-7}$
\end{tabular}

In Fig. 6 the refractive index profile measured in $3 \mu \mathrm{m}$ thick layers of $6 \mathrm{CHBT}$ and W602 initially twisted has been shown. The behaviour of turning points exhibits that 6 CHBT is anchored stronger than W602 but the nematic mixture is more stiff than 6CHBT.

Such result is in accordance with the data from Tables I and II. The main component (about $40 \%$ of weight in $20^{\circ} \mathrm{C}$ ) of W602 is $6 \mathrm{CHBT}$. Other components, whose amounts are similar, have long molecules with a bridge $\mathrm{CH}_{2}-\mathrm{CH}_{2}$. The third component of W602 mixture is a non-polar compound. We do suppose that the non-polar components produce the weakening of the surface interactions. But this conclusion is only a hypothesis that should be verified in wider investigations. The presented results seem to allow one to create such a hypothesis.

\section{Conclusions}

A method for elastic properties of liquid crystals has been presented. The results for 6CHBT obtained here are close to that referred earlier (see Table III). It is a kind of verification for the proposed method. The elastic constants for nematic mixture W602 have been presented for the first time. A comparison of both investigated substances drives to a hypothesis of the weakening of surface anchoring in the presence of non-polar components of the nematic mixture produces. Following the Faeti conclusion about the renormalization of anchoring energy a new procedure for $k_{13}$ and $W_{s}$ calculation has been successfully done for both examined substances.

The investigations have been based on the direct refractive index profile measurement which is a very promising method.

This work is supported by the Committee for Scientific Research grant 7 T08C 00712.

\section{References}

[1] Iam-Choon Khoo, Shin-Tson Wu, Optics and Nonlinear Optics of Liquid Crystals, World Scientific Publ., Singapore 1993 (and references therein).

[2] J. Kędzierski, Elasticity, Diamagnetic, Susceptibility and Interaction of Nematies with Substrate, WAT, Warszawa 1994, p. 87, 132 (in Polish).

[3] J.T. Gleeson, Liq. Cryst. 20, 453 (1996).

[4] R. Buchecker, M. Schadt, Mol. Cryst. Liq. Cryst. 149, 359 (1987).

[5] A. Walczak, A. Kieżun, E. Nowinowski-Kruszelnicki, L.R. Jaroszewicz, Electron. Telecommun. Q. 42, 335 (1996). 
[6] A. Walczak, E. Nowinowski-Kruszelnicki, A. Kieżun, SPIE Proc. 3318, 344 (1997).

[7] K.R. Welford, J.R. Sambles, Liq. Cryst. 2, 91 (1987).

[8] J.R. Sambles, Fuzi Yang, G.W. Bradberry, Ferroelectrics 179, 257 (1996).

[9] D.J. Mikulin, D.A. Coley, J.R. Sambles, Liq. Cryst. 22, 301 (1997).

[10] C.R. Lavers, J.R. Sambles, Liq. Cryst. 8, 577 (1990).

[11] E.L. Wood, J.R. Sambles, SPIE Proc. 1845, 379 (1992).

[12] R.A. Stevens, J.R. Sambles, Liq. Cryst. 18, 413 (1995).

[13] S.J. Elston, J.R. Sambles, M.G. Clark, J. Mod. Optics 36, 1019 (1989).

[14] Fuzi Yang, J.R. Sambles, G.W. Bradberry, J. App. Phys. 78, 2187 (1995).

[15] S.J. Elston, Liq. Cryst. 9, 769 (1991).

[16] A. Walczak, Bulletin WAT 3, 172 (1997).

[17] R. Ulrich, P.K. Tien, J. Opt. Soc. Am. 60, 1325 (1970).

[18] A. Snyder, J.D. Love, Optical Waveguide Theory, Chapman and Hall, London 1987, p. 390.

[19] R. Buchecker, M. Schadt, Mol. Cryst. Liq. Cryst. 149, 359 (1987).

[20] R. Dąbrowski, J. Dziaduszek, T. Szczuciński, Mol. Cryst. Liq. Cryst. 124, 241 (1984).

[21] J. Legras, Methodes et Techniques de l'Analyse Numerique, Dunod, Paris 1971, p. 72.

[22] Ch.L. Lawson, R.J. Hanson, Solving Least Squares Problems, Prentice-Hall, Engelwood Cliffs 1974, p. 45, 28.

[23] A. Rapini, M. Papoular, J. Phys. Collog. 30, C-4, 54 (1974).

[24] C. Rosenblatt, J. Phys. 45, 1087 (1984); H. Yokoyama, H.A. Sprang, J. Appl. Phys. 57, 4520 (1985).

[25] H. Yokoyama, S. Kobayashi, H. Kamei, J. Appl. Phys. 61, 4501 (1987).

[26] A.L. Alexe-Ionescu, G. Barberi, G. Barbero, M. Giocondo, Phys. Rev. E 49, 5378 (1994).

[27] S. Faeti, Mol. Cryst. Liq. Cryst. 179, 217 (1990).

[28] V.M. Pergamenschik, Phys. Rev. E 48, 1254 (1993).

[29] J. Nehring, A. Saupe, J. Chem. Phys. 54, 337 (1971). 